\title{
Synthesis of Isotopically-Labeled Graphite Films by Cold-Wall Chemical Vapor Deposition and Electronic Properties of Graphene Obtained from Such Films
}

\author{
Weiwei Cai ${ }^{1}(\bowtie)$, Richard D. Piner ${ }^{1}$, Yanwu Zhu ${ }^{1}$, Xuesong $\mathrm{Li}^{1}$, Zhenbing Tan $^{2}$, Herman Carlo Floresca ${ }^{3}$, \\ Changli Yang ${ }^{2}$, Li Lu', M. J. Kim³ ${ }^{3}$ Rodney S. Ruoff ${ }^{1}(\square)$ \\ ${ }^{1}$ Department of Mechanical Engineering and the Texas Materials Institute, University of Texas at Austin, Austin, TX 78712, USA \\ ${ }^{2}$ Institute of Physics, Chinese Academy of Sciences, Beijing 100190, China \\ ${ }^{3}$ Department of Materials Science and Engineering, University of Texas at Dallas, Richardson, TX 75083, USA
}

Received: 12 June 2009 / Revised: 19 August 2009 / Accepted: 22 August 2009

CTsinghua University Press and Springer-Verlag 2009. This article is published with open access at Springerlink.com

\begin{abstract}
We report the synthesis of isotopically-labeled graphite films on nickel substrates by using cold-wall chemical vapor deposition (CVD). During the synthesis, carbon from ${ }^{12} \mathrm{C}$ - and ${ }^{13} \mathrm{C}$-methane was deposited on, and dissolved in, a nickel foil at high temperature, and a uniform graphite film was segregated from the nickel surface by cooling the sample to room temperature. Scanning and transmission electron microscopy, microRaman spectroscopy, and X-ray diffraction prove the presence of a graphite film. Monolayer graphene films obtained from such isotopically-labeled graphite films by mechanical methods have electron mobility values greater than $5000 \mathrm{~cm}^{2} \cdot \mathrm{V}^{-1} \cdot \mathrm{s}^{-1}$ at low temperatures. Furthermore, such films exhibit the half-integer quantum Hall effect over a wide temperature range from $2 \mathrm{~K}$ to $200 \mathrm{~K}$, implying that the graphite grown by this cold-wall CVD approach has a quality as high as highly oriented pyrolytic graphite (HOPG). The results from transport measurements indicate that ${ }^{13} \mathrm{C}$-labeling does not significantly affect the electrical transport properties of graphene.
\end{abstract}

\section{KEYWORDS}

Chemical vapor deposition (CVD), isotopically-labeled graphite, graphene

\section{Introduction}

Carbon has two stable isotopes, ${ }^{12} \mathrm{C}$ and ${ }^{13} \mathrm{C}$, with natural abundances of $98.9 \%$ and $1.1 \%$, respectively. In 1990, researchers at General Electric (GE) succeeded in growing large, high-quality singlecrystal diamonds across the entire range of isotopic compositions [1]. The subsequent discovery that the room-temperature thermal conductivity of nearly pure ${ }^{12} \mathrm{C}$ diamond is over $50 \%$ greater than that of natural diamond generated further scientific and technological interest [2]. Graphene-based materials exhibit fascinating electronic transport properties that sensitively depend on the number of stacked graphene layers [3]. These properties suggest the possibility of high speed (THz) electronic devices,

Address correspondence to Rodney S. Ruoff, r.ruoff@mail.utexas.edu; Weiwei Cai, wc4943@mail.utexas.edu 
spintronic devices, sensors, memory devices for massive data storage, and new approaches for thermal management, among others. Furthermore, isotopically-labeled graphite provides an important degree of freedom for both fundamental studies and applications.

Solid-state nuclear magnetic resonance (SSNMR) provides a powerful method to study the chemical structure of graphene-based materials, which are amorphous or vary in chemical composition [4]. However, ${ }^{13} \mathrm{C}$ NMR is much less sensitive to carbon than ${ }^{1} \mathrm{H}$ NMR is to hydrogen because of its low abundance. Recently, the successful synthesis of ${ }^{13} \mathrm{C}$-enriched graphite allowed ${ }^{13} \mathrm{C}-{ }^{13} \mathrm{C}$ connectivities to be detected by NMR [5].

On the other hand, graphite formation on the surface of metals, particularly transition metals, has been studied for a long time [5-11]. The "metallurgical model" explains the formation of graphite in such systems. Since carbon is being produced on the metal surface by decomposing hydrocarbon gas, the concentration gradient causes it to diffuse into the metal, normal to the surface. The solubility of carbon in some metals increases with temperature. For example, nickel dissolves $1.3 \%, 1.5 \%$, and $1.8 \%$ of carbon at 1000,1050 , and $1100{ }^{\circ} \mathrm{C}$, respectively. A fraction of the carbon atoms in a solid solution in a metal at elevated temperatures, are known to precipitate as a graphite film upon cooling. Here, we present a simple cold-wall chemical vapor deposition (CVD) method for depositing and segregating a high quality isotopically-labeled graphite film on a nickel foil and measurements of the transport properties of graphene obtained from such graphite films.

\section{Experimental}

The home-made CVD apparatus [5] used to grow isotopically-labeled graphite consists in part of a twoway, a three-way, and a four-way standard stainlesssteel International Organization for Standardization (ISO) cross. A $5 \mathrm{~mm} \times 50 \mathrm{~mm}$ polycrystalline nickel foil $0.05 \mathrm{~mm}$ in thickness (Alfa Aesar, annealed, 99.9\% (metals basis)) was mounted at the central position of the chamber with two Mo clamps and this foil was held under tension by a copper spring.
The substrate was resistively heated to a high temperature by applying a current through two water-cooled electrical feedthroughs. Three precision stainless steel bars were mounted on a flange and used to hold the electrodes and copper plates. The entire outer wall of the chamber was surrounded by soft copper tubing for water cooling. ${ }^{13} \mathrm{CH}_{4}(>99.95 \%$; Cambridge Isotopes Laboratories, Inc.), 'normal' methane (unlabeled, henceforth simply referred to as "methane"), and argon (both supplied by Airgas, 99.999\%) were introduced through valves connected to the chamber. Three of them, connected by a small " $\mathrm{T}$ " were set up and the ratio of ${ }^{13} \mathrm{CH}_{4}$ and methane introduced into the chamber was controlled by opening and closing the valves in turn. The typical current used during growth was $20 \mathrm{~A}$ at about $6 \mathrm{~V}$ bias (Lambda power supply, model Genesys $3.3 \mathrm{~kW}$ ). The growth temperature was monitored by a potentiometer type optical pyrometer (Leeds \& Northrup Co. model No. 8622). Pressure was measured with a thermocouple gauge (Edwards BOC, model ACT-E). The vacuum pump was a model RV3 from Edwards BOC.

The isotopic composition of the as-produced graphite was determined with a modified CAMECA ims3f secondary ion mass spectrometer (SIMS). Data from mass spectrometry were used to confirm the validity of confocal micro-Raman spectroscopy (WITec Alfa 300), as a means to measure ${ }^{13} \mathrm{C} /{ }^{12} \mathrm{C}$ ratios [5]. The excitation power was about $50 \mathrm{~mW}$ at wavelength of $5320 \AA$. The laser beam was focused on the sample surface with a 100X microscope objective. The peak frequency shifts were used to determine the ${ }^{13} \mathrm{C} /{ }^{12} \mathrm{C}$ ratios [5]. The quality of graphite samples was ascertained from lack of a D peak in the Raman spectra, as discussed below.

In order to obtain structural information for our graphite film, scanning electron microscopy (SEM, FEI Quanta-600), high-resolution transmission electron microscopy (HRTEM, JEM-2100F) and X-ray diffraction (XRD, Philips, APD3520) were performed. The transmission electron microscopy (TEM) sample was prepared using the focused ion beam (FIB, Nova 200 NanoLab) lift-out method, where the sample was created from the bulk, lifted out by a nanomanipulator and welded to a copper grid for 
viewing. To achieve a clear layered image of graphite films, the samples were tilted in the TEM to orient the layers parallel to the electron beam.

By using the mechanical exfoliation method, similar to that described in Ref. [3], the exfoliated monolayer graphene samples were extracted from as-produced isotopically-labeled graphite and transferred onto a $\mathrm{Si}$ substrate with a $300-\mathrm{nm} \mathrm{SiO}_{2}$ coating layer. Interference-induced color shifts allowed us to identify the number of graphene layers from optical images of the samples [12]. Once a proper graphene sample was selected, it was further tailored to a Hall-bar by electron beam lithography (EBL Raith 150) and oxygen plasma etching, with poly(methyl methacrylate) (PMMA) as the resist. Ohmic electrodes were also defined by EBL, followed by 50-nm-thick Pd evaporation and the lift-off process. Transport measurements were performed on a Physical Property Measurement System (PPMS, Quantum Design) which can provide a base temperature of $2.0 \mathrm{~K}$ and a magnetic field up to $14 \mathrm{~T}$. The sample was annealed in situ for $1 \mathrm{~h}$ at $T=380 \mathrm{~K}$ before cooling down. The magnetoresistance $R_{x x}$ and Hall resistance $R_{x y}$ were measured using standard low-frequency $(30.9 \mathrm{~Hz})$ lock-in techniques, with a small current of $10 \mathrm{nA}$. The carrier density of the Hall-bar device was tuned by a gate voltage $V_{\mathrm{g}}$ applied to the degenerately-doped Si substrate.

\section{Results and discussion}

During carbon deposition, the nickel foil was

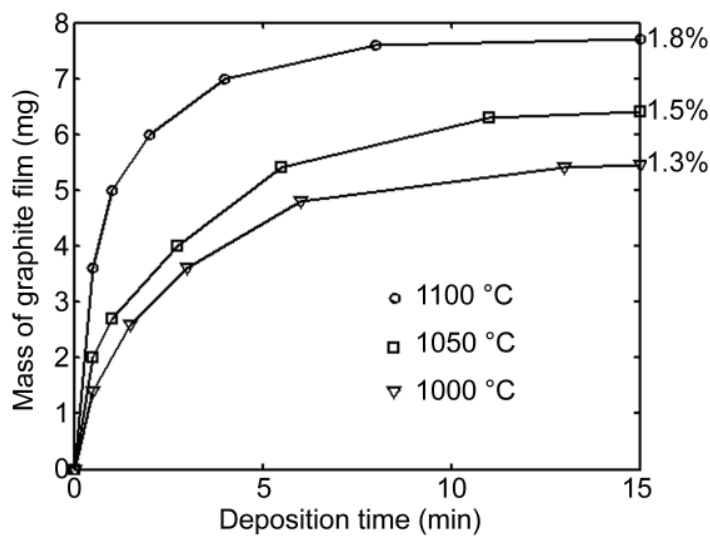

(a) resistively heated to a temperature between $1000{ }^{\circ} \mathrm{C}$ and $1100{ }^{\circ} \mathrm{C}$. Figure 1 (a) is a plot of the mass of graphite formed on the nickel foil as a function of deposition time and temperature. The mass of graphite was measured after growth, by subtracting the mass of nickel foil measured before graphite deposition. There is a rapid increase in the graphite film mass for the first 2 min of exposure to methane, followed by a slower increase for longer exposures, and no further increase after about $15 \mathrm{~min}$. The atomic percentages of graphite and nickel are in good agreement with the prediction from the $\mathrm{C}-\mathrm{Ni}$ phase diagram (see Fig. 1(b)). This result indicates that the amounts of graphite are dependent on the solubility of carbon in nickel at high temperature as discussed above.

Raman spectroscopy is widely used to probe carbon materials to distinguish different types of microstructure. Some authors have reported that a decrease in the relative intensity of the $\mathrm{D}$ band corresponds to an increase in the graphite crystal size $[13,14]$. The $D$ band scattering from our sample (Fig. 2(a)) is so small that it is undetectable. Since the frequencies of Raman modes are inversely proportional to the square root of the atomic mass, with the assumption that the respective bond force constants in the ${ }^{12} \mathrm{C}$ and ${ }^{13} \mathrm{C}$ graphite are equal the frequencies of the graphite can be derived as

$$
\omega=\omega_{0} \times \sqrt{m_{12} / m^{*}}
$$

where $\omega_{0}$ is the Raman mode frequency of ${ }^{12} \mathrm{C}$ graphite, $m_{12}$ is the atomic mass of ${ }^{12} \mathrm{C}$, and $m^{*}$ is the

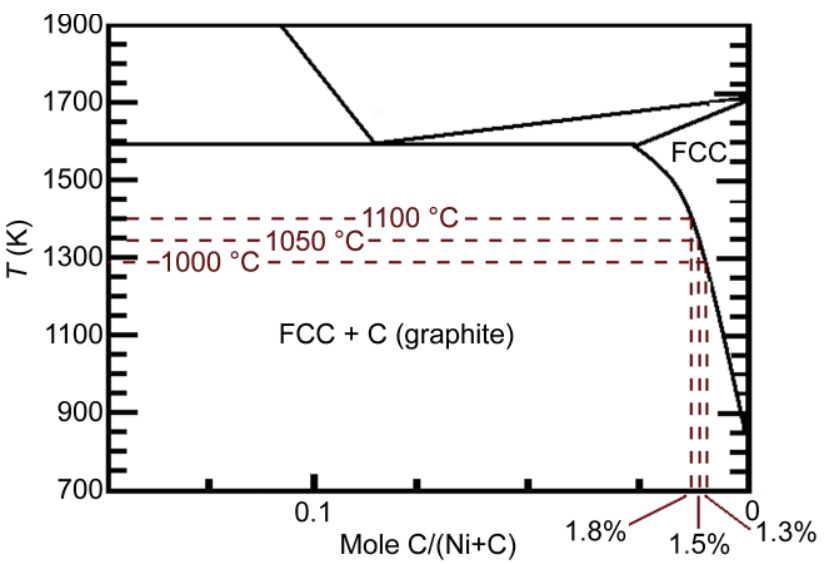

(b)

Figure 1 (a) The mass of graphite film formed on a nickel foil as a function of deposition time at three different deposition temperatures. (b) Binary phase diagram of $\mathrm{Ni}-\mathrm{C}$ alloy, where $\mathrm{FCC}$ represents face-centered cubic 
average atomic mass of the ${ }^{12} \mathrm{C}$ and ${ }^{13} \mathrm{C}$ present [15]. Figure 2(b) shows the relationship observed between the wavenumber of the $\mathrm{G}$ band and the ${ }^{13} \mathrm{C} /{ }^{12} \mathrm{C}$ ratio. The Raman frequencies shift from $\sim 1582 \mathrm{~cm}^{-1}$ for natural abundance to $\sim 1522 \mathrm{~cm}^{-1}$, for an enrichment of $99.5 \%{ }^{13} \mathrm{C}$ (measured by mass spectrometry [15]). There is good agreement between the frequency shift and the square root of the atomic mass (Eq. 1). This result indicates that the ${ }^{12} \mathrm{C}$ and ${ }^{13} \mathrm{C}$ atoms are mixed randomly at the atomic scale.

SEM and high resolution TEM were used to investigate the structure of the graphite films. The

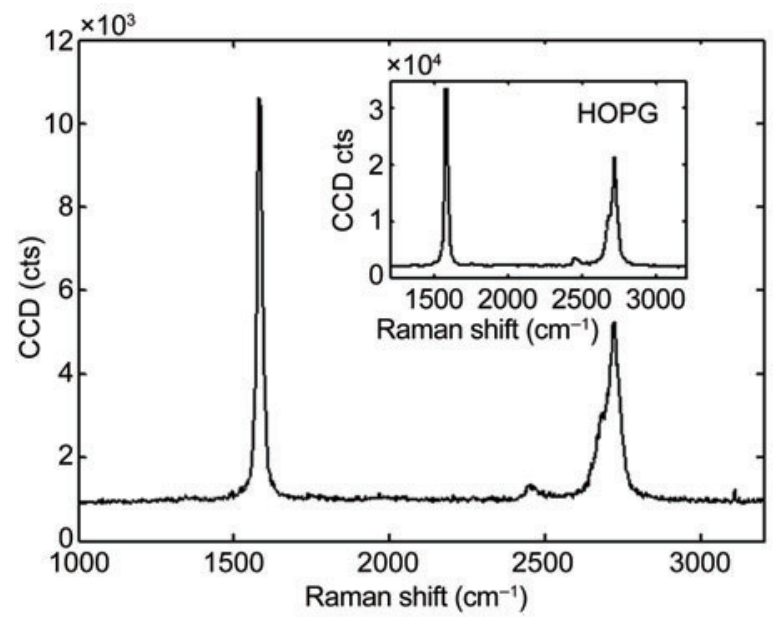

(a)
SEM images (Fig. 3(a)) show the morphology of typical graphite films. The films formed on the nickel foils appeared very smooth and continuous. The smooth surface areas are separated from each other by wrinkles that are likely caused by the different thermal expansion coefficients of graphite films and the nickel foil. A typical smooth surface region is about $2-\mu \mathrm{m}$ across, and the typical wrinkle height obtained by TEM images is about $100 \mathrm{~nm}$. Figure 3(b) shows a cross-section image of a 300-nmthick graphite film with a typical wrinkle in low magnification. A high magnification image (Fig.

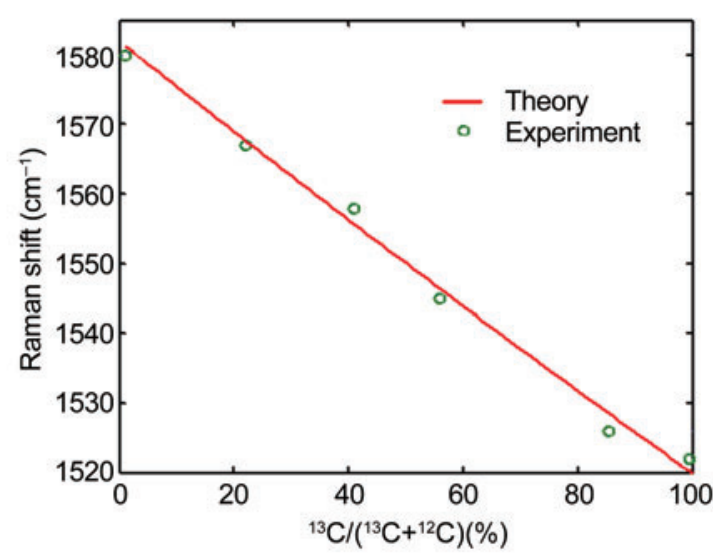

(b)

Figure 2 (a) Raman spectra of HOPG (inset) and of synthetic graphite on nickel. (b) Shift of the G band frequency as a function of increasing percentage of ${ }^{13} \mathrm{C}$, as determined by SIMS [15]. The theoretical curve was obtained using Eq. 1
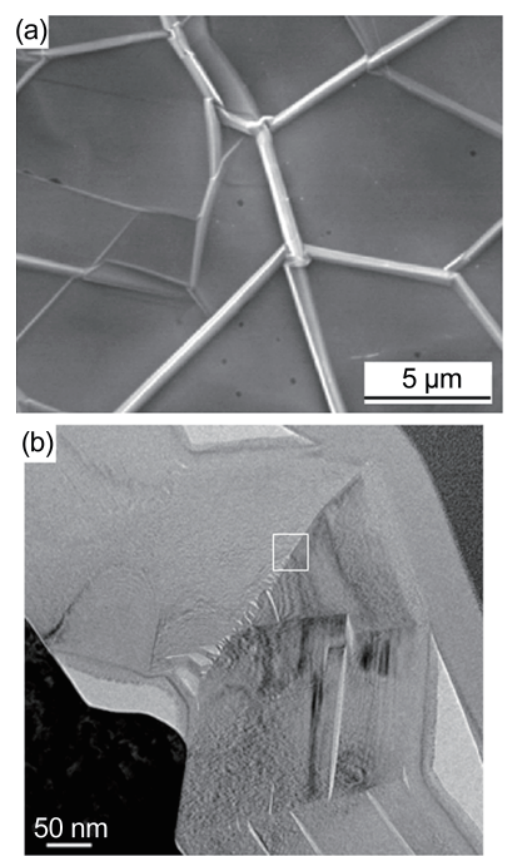

Figure 3 (a) SEM image of isotopically-labeled graphite film on nickel foil. TEM images of a cross-section in low magnification (b) and high magnification (c). In (b), the white rectangule shows the position of (c)

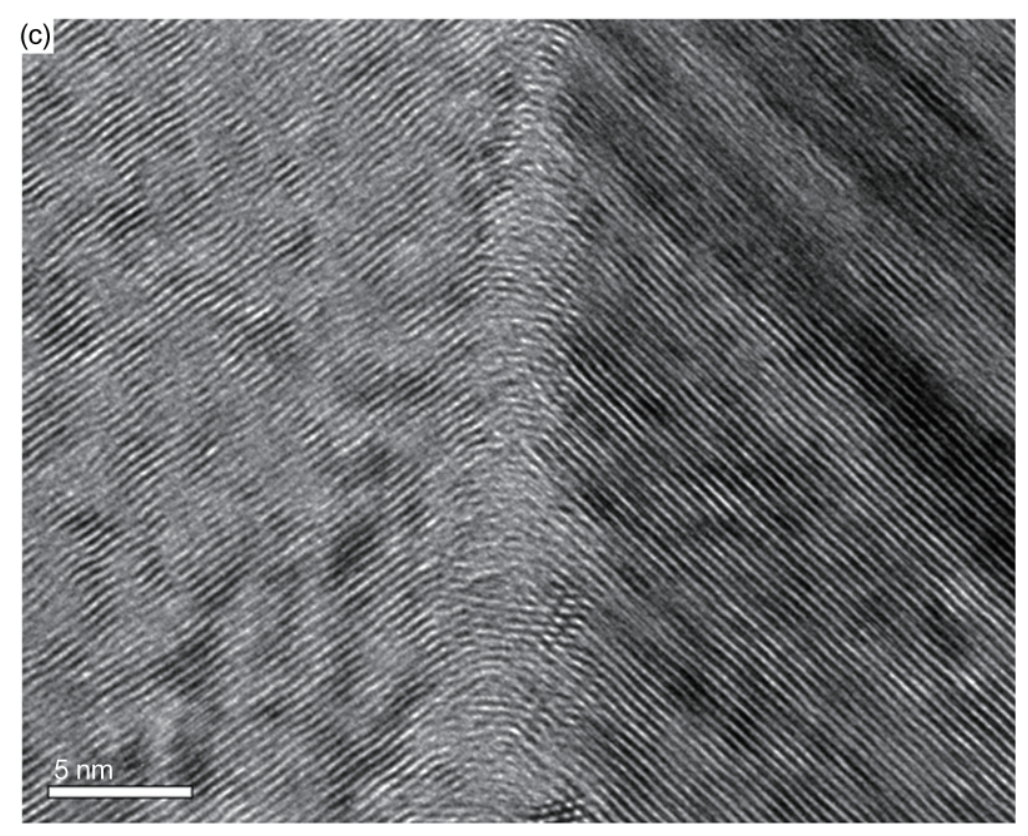


3(c)) clearly shows the perfect layer structure and the $d$-spacing of $3.35 \AA$. The layers on the right side of Fig. 3(c) are closely parallel to the electron beam, showing distinct layers, while the layers on the left are further from being parallel. The structure of the graphite films was also studied by XRD, where the diffractogram (Fig. 4) shows sharp (002) and (004) reflection peaks of the graphite film, removed from the nickel substrate. The fitted graphite $d$-spacing is $3.343 \AA \pm 0.005 \AA$, in good agreement with the results of cross-section TEM characterization.

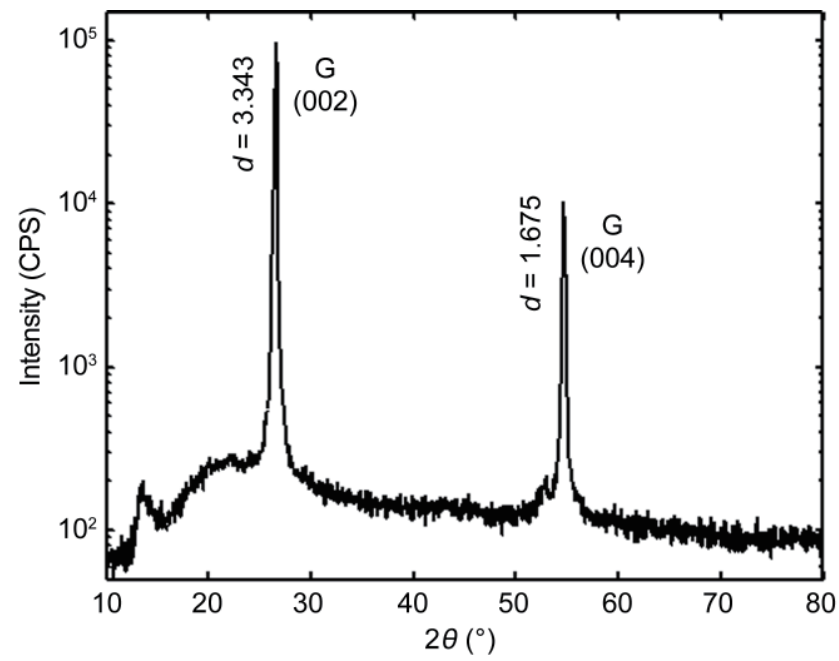

Figure 4 XRD pattern of isotopically-labeled graphite film

The electron mobility in graphene is critical for microelectronic applications. In order to measure the intrinsic mobility of the electrons, isotopic graphenes were extracted from as-produced isotopically-labeled graphite onto a $\mathrm{Si}$ substrate by using a mechanical exfoliation method [3]. The SEM image of an exfoliated graphene Hall-bar device is shown in the lower inset of Fig. 5. The atomic percentage of ${ }^{13} \mathrm{C}\left({ }^{13} \mathrm{C} /\left({ }^{12} \mathrm{C}+{ }^{13} \mathrm{C}\right)\right)$ in this sample is $46 \%$. Electrical measurement at low temperatures shows that the electron mobility is about $5000 \mathrm{~cm}^{2} \cdot \mathrm{V}^{-1} \cdot \mathrm{s}^{-1}$ at a carrier density of $\sim 5 \times 10^{12} \mathrm{~cm}^{-2}$. Figure 5 shows longitudinal $\left(R_{x x}\right)$ and transverse $\left(R_{x y}\right)$ magnetoresistances measured as a function of gate voltage $V_{\mathrm{g}}$ at a fixed magnetic field $B=14 \mathrm{~T}$. For this graphene sample, the neutral or Dirac point occurs at $V_{\mathrm{g}} \approx 10 \mathrm{~V}$, as indicated by the dominant strongest peak in $R_{x x}$. Corresponding to minima in $R_{x x}$ quantized plateaus around the Landau level filling factor $v= \pm 2, \pm 6, \pm 10$ are clearly observed in $R_{x y}$ exhibiting the characteristic half- integer quantum Hall effect of a single-layered graphene [16]. The half-integer quantum Hall effect can be observed up to $T=200 \mathrm{~K}$, as shown in the upper inset of Fig. 5. This result indicates that the quality of the synthetic graphite film is comparable to that of highly oriented pyrolytic graphite (HOPG) and that the ${ }^{13} \mathrm{C}$ labeling does not significantly decrease the mobility of electrons in isotopicallylabeled graphene.

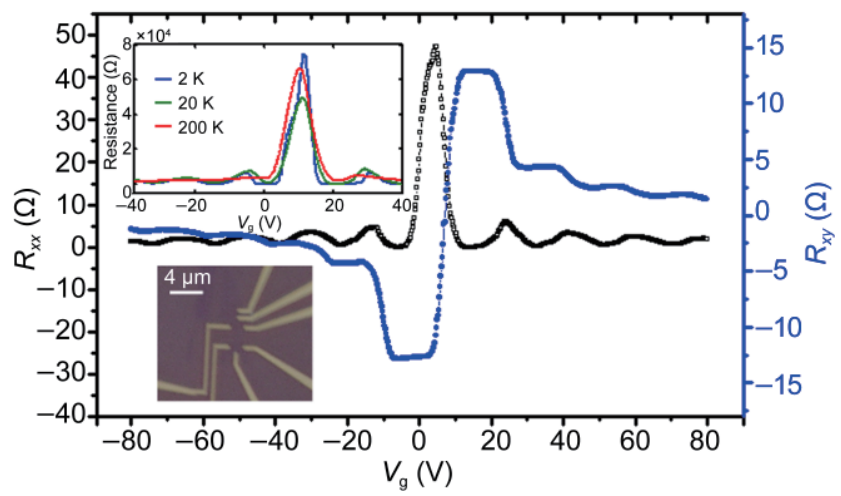

Figure 5 Electrical transport of an exfoliated isotopically-labeled graphene device that shows the half-integer quantum Hall effect. Lower inset: a SEM image of the monolayer graphene Hall-bar device; Upper inset: electrical transport of the device at selected temperatures of $2.0 \mathrm{~K}, 20 \mathrm{~K}$, and $200 \mathrm{~K}$ (from another cool down)

\section{Conclusions}

Isotopically-labeled graphite films have been grown on nickel substrates from a ${ }^{13} \mathrm{CH}_{4}$ and ${ }^{12} \mathrm{CH}_{4}$ gas mixture by cold-wall CVD. Investigation by SEM, TEM, XRD, and micro-Raman spectroscopy prove the presence of a well-ordered graphite film on the Ni. At low temperatures, monolayer graphene films cleaved from isotopically-labeled graphite by mechanical exfoliation show an electron mobility greater than $5,000 \mathrm{~cm}^{2} \cdot \mathrm{V}^{-1} \cdot \mathrm{s}^{-1}$ and exhibit the half-integer quantum Hall effect, implying that the graphite grown by coldwall CVD is as high quality as HOPG and that the enrichment of ${ }^{13} \mathrm{C}$ does not significantly decrease the electron mobility.

\section{Acknowledgements}

This work was supported by The University of Texas at Austin and by the Texas Nanotechnology Research Superiority Initiative, Southwest Nanotechnology Institute (TNRSI) / SWAN. 


\section{References}

[1] Banholzer, W. F.; Anthony, T. R.; Gilmore, R. In Proceedings of the Second International Conference on New Diamond Science and Technology; Materials Research Society: Pittsburgh, 1990; pp 857.

[2] Anthony, T. R.; Banholzer, W. F.; Fleischer, J. F.; Wei, L. H.; Kuo, P. K.; Thomas, R. L.; Pryor, R. W. Thermal-diffusivity of isotopically enriched C-12 diamond. Phys. Rev. B 1990, 42, 1104-1111.

[3] Novoselov, K. S.; Geim, A. K.; Morozov, S. V.; Jiang, D.; Zhang, Y.; Dubonos, S. V.; Grigorieva, I. V.; Firsov, A. A. Electric field effect in atomically thin carbon films. Science 2004, 306, 666-669.

[4] Stankovich, S.; Dikin, D. A.; Piner, R. D.; Kohlhaas, K. A.; Kleinhammes, A.; Jia, Y.; Wu, Y.; Nguyen, S. T.; Ruoff, R. $S$. Synthesis of graphene-based nanosheets via chemical reduction of exfoliated graphite oxide. Carbon 2007, 45, 1558-1565.

[5] Cai, W. W.; Piner, R. D.; Stadermann, F. J.; Park, S.; Shaibat, M. A.; Ishii, Y.; Yang, D. X.; Velamakanni, A.; An, S. J.; Stoller, M.; An, J. H.; Chen, D. M.; Ruoff, R. S. Synthesis and solid-state NMR structural characterization of C-13-labeled graphite oxide. Science 2008, 321, 1815 $-1817$.

[6] Robertson, S. D. Graphite formation from low temperature pyrolysis of methane over some transition metal surfaces. Nature 1969, 221, 1044-1046.

[7] Fedoseev, D. V.; Vnukov, S. P.; Derjaguin, B. V. Physicochemical theory of graphite growth from hydrocarbons. Carbon 1979, 17, 453-458.

[8] Fujita, D.; Homma, T. Surface precipitation of graphite layers on carbon-doped nickel and their stabilization effect against chemisorption and initial oxidation. Surf. Interf. Anal. 1992, 19, 430-434.

[9] Obraztsov, A. N.; Obraztsova, E. A.; Tyurnina, A. V.; Zolotukhin, A. A. Chemical vapor deposition of thin graphite films of nanometer thickness. Carbon 2007, 45, 2017-2021.

[10] Yu, Q. K.; Lian, J.; Siriponglert, S.; Li, H.; Chen, Y. P.; Pei, S. S. Graphene segregated on Ni surfaces and transferred to insulators. Appl. Phys. Lett. 2008, 93, 113103.

[11] Kim, K. S.; Zhao, Y.; Jang, H.; Lee, S. Y.; Kim, J. M.; Ahn, J. H.; Kim, P.; Choi, J. Y.; Hong, B. H. Large-scale pattern growth of graphene films for stretchable transparent electrodes. Nature 2009, 457, 706-710.

[12] Jung, I.; Pelton, M.; Piner, R.; Dikin, D. A.; Stankovich, S.; Watcharotone, S.; Hausner, M.; Ruoff, R. S. Simple approach for high-contrast optical imaging and characterization of graphene-based sheets. Nano Lett. 2007, 7, 3569-3575.

[13] Knight, D. S.; White, W. B. Characterization of diamond films by Raman-spectroscopy. J. Mater. Res. 1989, 4, 385 $-393$.

[14] Nemanich, R. J.; Solin, S. A. 1st-order and 2nd-order Raman-scattering from finite-size crystals of graphite. Phys. Rev. B 1979, 20, 392-401.

[15] Fan, S. S.; Liu, L.; Liu, M. Monitoring the growth of carbon nanotubes by carbon isotope labelling. Nanotechnology 2003, 14, 1118-1123.

[16] Zhang, Y.; Tan, Y.; Stomer, H. L.; Kim, P. Experimental observation of the quantum Hall effect and Berry's phase in graphene. Nature 2005, 438, 201-204. 